\title{
Cardiovascular Circulation and Hepatic Perfusion of Pigs in 4-Dimensional Films Evaluated by 256-Slice Cone-Beam Computed Tomography
}

\author{
Nobusada Funabashi, MD; Katsuya Yoshida, MD; Hiroyuki Tadokoro, MD*; \\ Keiichi Nakagawa, MD; Nobuyuki Komiyama, MD; Kenichi Odaka, MD*; \\ Takanori Tsunoo, PhD*; Shinichiro Mori, MSc*; Shuji Tanada, MD*; \\ Masahiro Endo, $\mathrm{PhD} *$; Issei Komuro, $\mathrm{MD}$
}

\begin{abstract}
Background In both cardiac and hepatic disorders it is desirable to accurately visualize the direction and scale of blood flow in the whole organ in pulsating 3-dimensional (D) images, which are known as 4-D images.

Methods and Results The present study used 256-slice cone-beam computed tomography (CT) (Athena, Sony-Toshiba) at one rotation per second and a section thickness of $0.5 \mathrm{~mm}$ to show the dynamics of cardiovascular circulation and hepatic perfusion by contrast injection in 4-D films of pigs. Four pigs (20 kg each) were anesthetized with isoflurane. The distal tips of the catheters were positioned in the inferior vena cava (IVC) (pigs 1-3) and in the proper hepatic artery (pig 4). Volumetric scanning and injection of contrast material were started simultaneously and continued for $25 \mathrm{~s}$ with image reconstruction at 1-s intervals. In pigs 1-3, 4-D filming revealed the dynamics of cardiovascular circulation, first in the IVC, followed by the right ventricle and pulmonary artery, then the left ventricle, left atrium, pulmonary vein, and finally, the right heart disappeared and only the left heart and aorta remained visible. In pig 4, the hepatic arterial trees, followed by the venous trees, could be easily visualized in turn on the 4-D images.

Conclusions This technology successfully demonstrated cardiovascular circulation and hepatic perfusion in 4-D and will have clinical applicability. (Circ J 2005; 69: 585-589)
\end{abstract}

Key Words: 4-D perfusion cardiovascular circulation; Hepatic perfusion; 256-slice cone beam computed tomography

$\mathbf{I}^{\mathrm{n}}$ cardiovascular medicine, many subjects present with circulatory disorders, such as shunt flow in subjects with congenital shunt disease, or disorders of blood inflow into the left atrial appendage (LAA) in subjects with chronic atrial fibrillation 2,3 or left ventricular (LV) aneurysm seen in those with extensive anterior myocardial infarction, which may lead to thrombi in the LAA or LV aneurysm. Therefore, it is desirable to accurately visualize the direction and scale of the shunt flow or disorders of blood flow in the whole heart in pulsating 3-dimensional (D) images, which are known as 4-D images.

Similarly, in hepatic medicine, many subjects present with perfusion disorders such as liver cirrhosis or hepatocellular carcinomas and perfusion studies are performed to characterize the blood flow:-7 Again, in such cases it is desirable to continuously visualize all the hepatic arterial, capillary and venous trees in 4-D images.

In the studies using a prototype high-speed cone-beam computed tomography (CT) apparatus employing 256detector rows (Athena, Sony-Toshiba), an entire volume of

(Received November 30, 2004; revised manuscript received January 24, 2005; accepted February 7, 2005)

Department of Cardiovascular Science and Medicine, Chiba University Graduate School of Medicine, *National Institute of Radiological Sciences, Chiba, Japan

Mailing address: Nobusada Funabashi, MD, Department of Cardiovascular Science and Medicine, Chiba University Graduate School of Medicine, 1-8-1 Inohana, Chuo-ku, Chiba 260-8670, Japan. E-mail: nobusada@ma.kcom.ne.jp the thoracic part or liver can be imaged within a single gantry rotation (1 second per rotation) and a section thickness of $0.5 \mathrm{~mm}{ }^{8-10}$ Furthermore, maximum continuous $25 \mathrm{~s}$ scanning enables 4-D analysis. Although this CT technique does not involve electorcardiogram (ECG)-gated acquisition, the combination of its synchrony with volumetric data and reconstruction technique in which 1 scanning period is divided into a maximum of 100 phases, facilitates selection of the most static images and allows entire heart data acquisition without cardiac motion artifacts. In the present study we investigated the ability of this new technology to capture cardiovascular circulation and hepatic perfusion on 4-D dynamic volumetric images of pigs.

\section{Methods}

Four domestic pigs, weighing $20 \mathrm{~kg}$ each, were mechanically ventilated under isoflurane anesthesia. The distal tips of the catheters were positioned in the inferior vena cava (IVC) for the cardiovascular system (pigs 1-3) and in the proper hepatic artery (pig 4) for obtaining images of the hepatic perfusion. The heart rate of the pigs ranged from $70-80$ beats $/ \mathrm{min}$. The experiments were approved by the Animal Welfare Committee of the Institute, and were performed in compliance with the guidelines for the care and use of laboratory animals as described by the National Institutes of Health.

Scan conditions for the 256-slice cone-beam CT were: $120 \mathrm{kV}, 200 \mathrm{~mA}, 1.0 \mathrm{~s}$ exposure, $1.0 \mathrm{~s}$ gantry rotation time, 

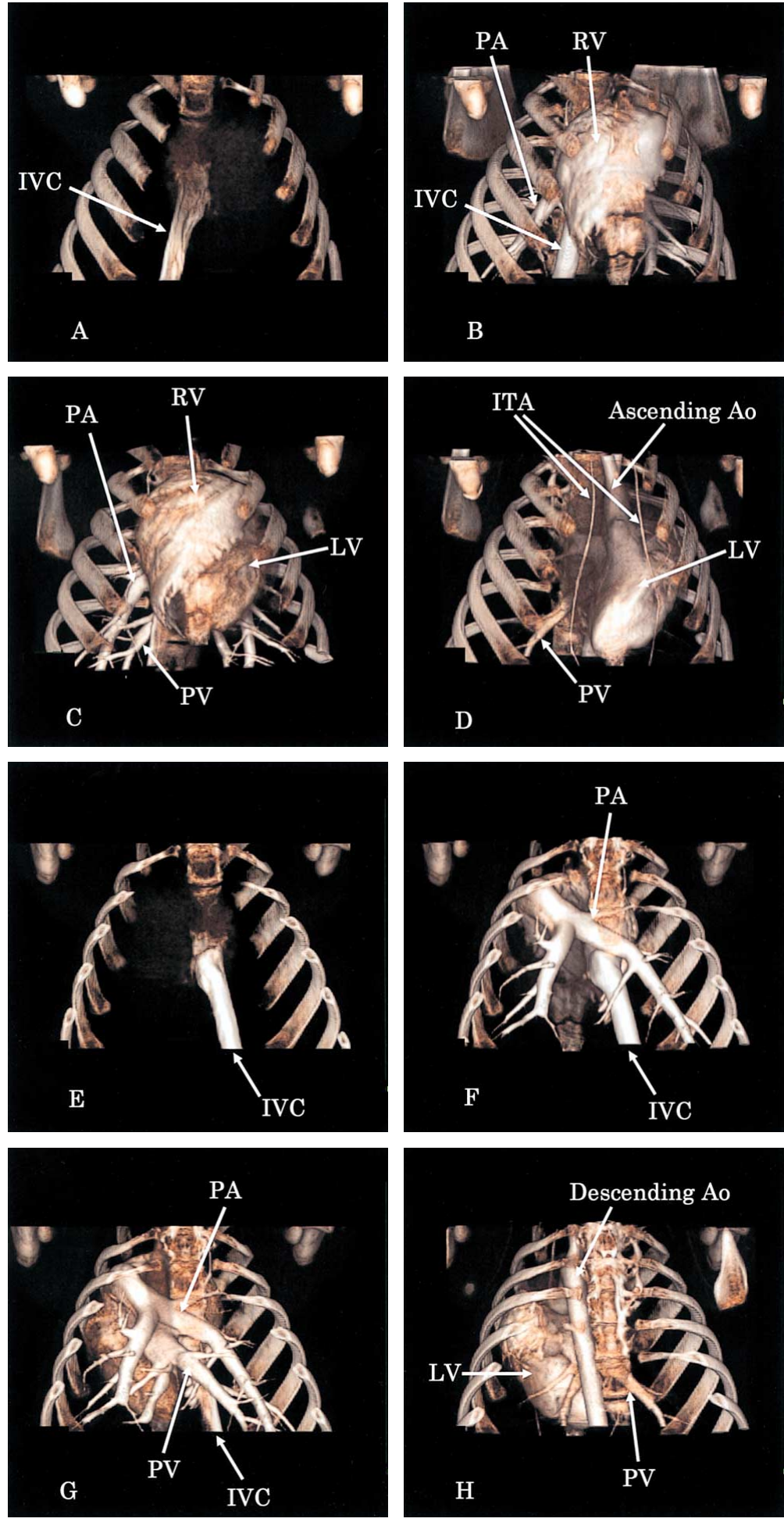

Fig 1. Volume-rendered reconstruction images of a porcine heart using 256-slice cone beam computed tomography represent the vessel lumens and the heart cavity filled with contrast material, with the surrounding ribcage observed from the anterior view (A-D) and posterior view $(\mathrm{E}-\mathrm{H})$, acquired after injection of contrast at $2 \mathrm{~s}(\mathrm{~A}, \mathrm{E}), 5 \mathrm{~s}(\mathrm{~B}, \mathrm{~F}) 12 \mathrm{~s}(\mathrm{C}, \mathrm{G})$, and $17 \mathrm{~s}$ $(\mathrm{D}, \mathrm{H})$. At $2 \mathrm{~s}$ after injection of the contrast material, only the inferior vena cava (IVC) could be observed $(\mathrm{A}, \mathrm{E})$; at $5 \mathrm{~s}$, the right ventricle (RV), pulmonary artery (PA) and IVC could be observed (B, F); at $12 \mathrm{~s}$, in addition to the RV and PA, the left ventricle (LV) and pulmonary vein $(\mathrm{PV})$ could be observed $(\mathrm{C}, \mathrm{G})$; but at $17 \mathrm{~s}$, the right heart disappeared and only the $\mathrm{LV}, \mathrm{PV}$, ascending aorta (Ao) and the right and the left internal thoracic arteries (ITA) could be observed (D, H).
$0.5 \mathrm{~mm}$ slice thickness, and $256 \times 0.5 \mathrm{~mm}$ slice collimation.

To begin, $10 \mathrm{ml}$ of the iodinated contrast material ( $300 \mathrm{mgI} / \mathrm{ml}$ ) was diluted with $40 \mathrm{ml}$ of saline and injected at a rate of $3 \mathrm{ml} / \mathrm{s}$ at the same time as scanning of the entire heart was begun. Scanning continued for $25 \mathrm{~s}$ and in that time the radiation dose was $100 \mathrm{mSV}$ !

The temporal resolution of this new CT is $1 \mathrm{~s}$ and the spatial resolution is approximately $1.0 \mathrm{~mm}$ (transverse) and $1.3 \mathrm{~mm}$ (longitudinal). Image reconstruction was performed at every 0.1-s interval and we selected the most static images around every 1-s interval after injection of contrast resulting in 25-phase volume data which were transferred to a workstation (Virtual Place Advance Plus, AZE, Japan).

\section{Results}

In pigs $1-3$, at $2 \mathrm{~s}$ after injection, the volume-rendered images showed only the IVC (Fig $1 \mathrm{~A}, \mathrm{E})$; at $5 \mathrm{~s}$, the right 

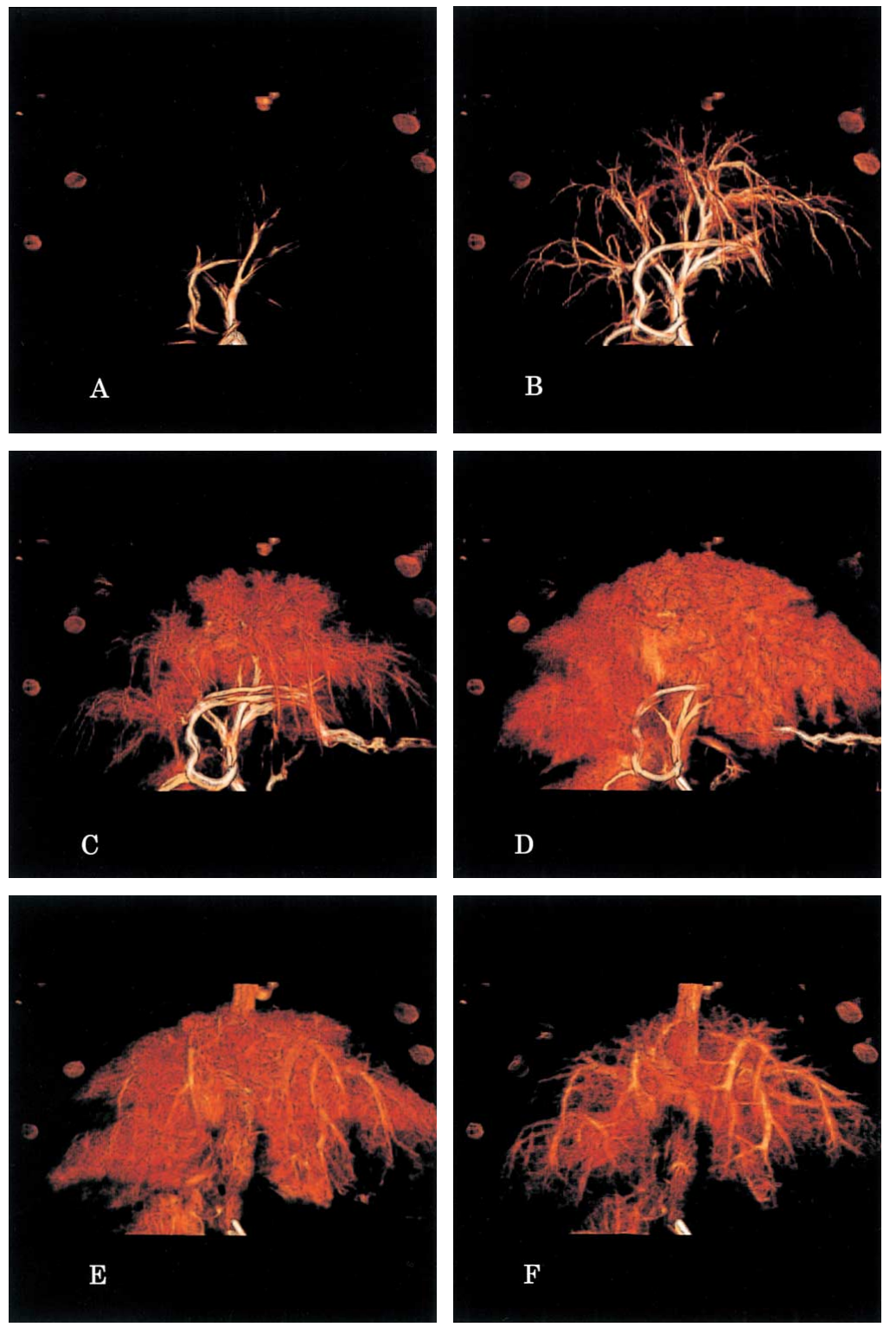

Fig 2. Volume-rendered reconstruction images of a porcine liver using 256-slice cone-beam computed tomography. (A, B) Hepatic arterial trees; (C, D) liver area supplied by blood capillaries within the hepatic arterial trees; (E, F) liver area supplied by blood capillaries beginning to disappear and the hepatic venous trees appearing. heart, including the right ventricle, the pulmonary artery, and the IVC could be seen (Fig 1B,F); at $12 \mathrm{~s}$, the LV, left atria (LA), including the LAA, and pulmonary vein (PV) could also be observed (Fig 1C,G). At 17 s, the right heart had disappeared and only the LV, LA and PV, ascending and descending aorta, and the right and left internal thoracic arteries were visible (Fig 1D,H). Finally the LAA images disappeared completely, followed by those of the $\mathrm{LV}$ and the dynamics of the cardiovascular circulation could be observed in 4-D filming (data not shown).

In pig 4 , at $3 \mathrm{~s}$ after injection, the volume-rendered images revealed only the hepatic arterial tree (Fig 2B); at $8 \mathrm{~s}$, the area supplied by capillaries could be observed (Fig 2D); at $15 \mathrm{~s}$, the hepatic arterial tree disappeared and the hepatic venous tree appeared (Fig 2F). These sequences could be visualized in 4-D filming, which allowed the hepatic perfusion to be easily observed (data not shown).

\section{Discussion}

Echocardiography, ${ }^{12,13}$ a flow pattern study of magnetic resonance imaging ${ }^{14}$ electron-beam CT,15,16 conventional left ventriculography ${ }^{17,18}$ and aortography have all been used to evaluate of cardiovascular blood flow, but they all use pulsating 2-D images only, which make it difficult to observe the 3-D spatial relationships circulation.

The 3-D images using ECG-gated multislice CT (MSCT) ${ }^{19}$ or electron-beam $\mathrm{CT}^{20-23}$ are constructed by building up data from the same cardiac phase (R-to-R interval) but with a different cardiac beat. However, these 3-D images do not allow any evaluation of the dynamics of blood flow.

The new 256-slice cone-beam CT with its combination of reconstruction techniques can successfully demonstrate the dynamics of the cardiovascular circulation system on 4-D films in which the configuration of the heart continued to the through plane without any gaps. In other words, this new prototype CT has a unique ability, synchrony, for acquiring images of the pulsating heart. Compared with a conventional 16-slice CT scanner with $0.75 \mathrm{~mm}$ slices, with this new 256-slice cone-beam CT, image noise, uniformity, and high contrast detectability are independent of the zaxis? Furthermore, the scanning mechanism can accommo- 
date a rotation speed of up to $0.5 \mathrm{~s} /$ rotation and ECG-gated acquisition will be possible in the next generation of scanner?

In the present MSCT, using retrospective ECG-gating acquisition or respiratory motion-gating acquisition, ${ }^{24,25}$ which use helical scanning with simultaneous recording of an ECG or respiratory motion signal, following acquisition, it is possible to obtain any volume data of the heart or lung at any cardiac or respiratory phase desired. Furthermore, using this system, it is possible to obtain maximum 100 volumetric data points divided into maximum 100 serial segments of one cardiac or respiratory phase such as 0,1 , 2....97, 98 and $99 \%$ of the R-to-R interval of the ECG or respiratory motion. Using serial maximum 100 phase volumetric data, it is also possible to obtain 4-D images on a workstation in which the motion of the heart or thoracic parts can be evaluated. Furthermore, this 256-slice conebeam CT could represent the dynamics of contrast material in 4-D images; although it is not possible with the present MSCT which so far has been developed as a 64-slice data acquisition system.

This new modality could have applications in the visualization of shunt flow and disorders of blood flow into the LAA and LV aneurysm, which in the latter case may lead to the formation of thrombi. It also may be able to provide visualization of myocardial blood flow when the temporal resolution improves. Gradation of the myocardial blood flow within the myocardium (ie, the difference in the blood flow in the endomyocardium and epicardium) may produce a large amount of information when myocardial ischemia develops.

In the present study, we also demonstrated hepatic perfusion combined with selective hepatic arterial injection and observed clear hepatic enhancement on the 4-D films. Some investigators have reported hepatic perfusion using 3-9 phases of volumetric data of the whole liver, or continuous acquisition for $30 \mathrm{~s}$ that covered parts of the liver in 4 slices with the use of time density curves ${ }^{5-7}$ However, the former technique does not use a sequential perfusion study, but is an intermittent perfusion study, and the latter technique is not an entirely volumetric only a partial volumetric hepatic perfusion study. To obtain 4-D images using the 256-slice cone-beam CT such as presented in this study, 2 techniques can be envisaged for the future.

One approach is to perform the same procedure as performed in pig 4 in this study using a combination of a selective angiography system with the CT system in which the CT acquisition with selective hepatic artery injection can be achieved without any movement of the patient. In fact, such a system is already in use ${ }^{26}$ and it would be possible to obtain 4-D images of the same hepatic enhancement presented in this study. Therefore, if the physician wishes to determine the area of liver supplied by the proper hepatic artery, or needs to obtain quantitative blood flow information in subjects with liver cirrhosis, or intends to evaluate the feeding artery of hepatocellular carcinoma, this technique provides useful information for devising the appropriate treatment strategy. In this study, because we placed the catheter in the proper hepatic artery, we could not evaluate the perfusion of the artery or the portal vein originating from the superior mesenteric artery or the splenic artery.

The other approach is non-invasive image acquisition using the new 256-slice cone-beam CT combined with intravenous injection. Even though with this method seg- mentation of liver enhancement cannot be performed, we can evaluate the 3-D perfusion of the whole liver non-invasively, which is not possible with 16-slice or even the latest 64-slice MSCT. Using this technique, continuous 25-s acquisitions can be performed, yielding quantitative hepatic perfusion information by using time density curves of the liver on 3-D images.

We describe here a novel prototype CT that has the advantage of whole heart and liver imaging in a single gantry rotation without ECG-gated acquisition. This technique may facilitate cardiovascular and liver diagnosis and may also find application with other organs such as the brain, kidney and peripheral vasculature.

One of the problems encountered with this new 256-slice cone-beam CT scanner is that in order to achieve a detailed 4-D analysis, the number of images that the workstation has to process is very large. For example, 1 volume data set has 256 images, which means that if an image is acquired from 10 phases per s over a total of $25 \mathrm{~s}$, the workstation has to process $64,000(256 \times 10 \times 25)$ images at one time in order to achieve a detailed 4-D analysis. At present even the most advanced workstation cannot process such a huge volume of images, but this problem may resolve within a few years as workstations become more sophisticated. Recently, increasing attention has been given to the dose of radiation received during in cardiac CT ${ }^{27}$ Specifically, the radiation dose for evaluating blood flow dynamics using the 256-slice cone-beam CT is estimated as $4 \mathrm{mSV} / \mathrm{s}$. Although it is better to keep the radiation dose to a minimum, it may be justifiable to perform such a procedure despite the radiation dose.

There is another type of $\mathrm{CT}$ available, a prototype flat-panel CT system (VCT, GE Global Research), with a $200-\mathrm{mm}$ detector panel and a cell pitch factor of $0.2 \mathrm{~mm}$, which also allows an entire organ to be imaged within a single gantry rotation ${ }^{28}$ Thus, in the near future, these new imaging modalities will likely become new diagnostic tools.

\section{Conclusion}

We successfully demonstrated the cardiovascular circulation and hepatic perfusion in 4-D using 256-slice conebeam CT. This imaging modality has clinical potential for the visualization and analysis of cardiovascular circulatory problems and hepatic perfusion disorders.

\section{Acknowledgment}

This work was supported in part by the 4D-CT research group at the National Institute of Radiological Sciences, Japan.

\section{References}

1. Funabashi N, Rubin GD. Qualitative blood flow differentiation: Depiction of a left to right cardiac shunt across a ventricular septal defect using electron-beam computed tomography. Jpn Circ J 2000; 64: $901-903$.

2. Nakanishi T, Hamada S, Takayama M, Naito H, Imakita S, Yamada $\mathrm{N}$, et al. A pitfall in ultrafast CT scanning for the detection of left atrial thrombi. J Comput Assist Tomogr 1993; 17: 42-45.

3. Kamiyama N, Koyama Y, Suetsuna R, Saito Y, Kaji S, Akasaka T, et al. Decreased left atrial appendage flow velocity with atrial fibrillation caused by negative inotropic agents. Circ J 2003; 67: 277-278.

4. Beppu S, Izumi S, Miyatake K, Nagata S, Park YD, Sakakibara H, et al. Abnormal blood pathways in left ventricular cavity in acute myocardial infarction: Experimental observations with special reference to regional wall motion abnormality and hemostasis. Circulation 1988; 78: $157-164$. 
5. Fuentes MA, Keith CJ, Griffiths M, Durbridge G, Miles KA. Hepatic haemodynamics: Interrelationships between contrast enhancement and perfusion on CT and Doppler perfusion indices. Br J Radiol 2002; 75: $17-23$.

6. Tsushima Y, Blomley MJ, Kusano S, Endo K. Measuring portal venous perfusion with contrast-enhanced CT: Comparison of direct and indirect methods. Acad Radiol 2002; 9: 276-282.

7. Cuenod CA, Leconte I, Siauve N, Resten A, Dromain C, Poulet B, et al. Early changes in liver perfusion caused by occult metastases in rats: Detection with quantitative CT. Radiology 2001; 218: 556561.

8. Mori S, Endo M, Tsunoo T, Kandatsu S, Tanada S, Aradate H, et al. Physical performance evaluation of a 256-slice CT scanner for fourdimensional imaging. Med Phys 2004; 31: 1348-1356.

9. Endo M, Tsunoo T, Kandatsu S, Tanada S, Aradate H, Saito Y. Fourdimensional computed tomography (4D CT) concepts and preliminary development. Radiat Med 2003; 21: 17-22.

10. Endo M, Mori S, Tsunoo T, Kandatsu S, Tanada S, Aradate H, et al Development and performance evaluation of the first model of $4 \mathrm{D}$ CT-scanner. IEEE Trans Nucl Sci 2003; 50: 1667-1671.

11. Leitz W, Axelsson B, Szendrö G. Computed tomography dose assessment: A practical approach. Radiat Protect Dosimetry 1995; 57: $377-380$.

12. Ito T, Suwa M, Imai M, Hozumi T, Tonari S, Kitaura Y. Acute effects of diltiazem on regional left ventricular diastolic filling dynamics in patients with hypertrophic cardiomyopathy as assessed by color kinesis. Circ J 2004; 68: 1035-1040.

13. Liu J, Murata K, Fujino T, Ueda K, Kimura K, Wada Y, et al. Effect of dobutamine on regional diastolic left ventricular asynchrony in patients with left ventricular hypertrophy. Circ J 2003; 67: 119-124.

14. Inoue $\mathrm{T}$, Watanabe $\mathrm{S}$, Sakurada $\mathrm{H}$, Ono K, Urano M, Hijikata Y, et al. Evaluation of flow volume and flow patterns in the patent false lumen of chronic aortic dissections using velocity-encoded cine magnetic resonance imaging. Jpn Circ J 2000; 64: 760-764.

15. Rumberger JA, Bell MR. Measurement of myocardial perfusion and cardiac output using intravenous injection methods by ultrafast (cine) computed tomography Invest Radiol 1992; 27: 40-46.

16. Ludman PF, Darby M, Tomlinson N, Poole-Wilson PA, Rees S. Cardiac flow measurement by ultrafast CT: Validation of continuous and pulsatile flow. J Comput Assist Tomogr 1992; 16: 795-803.

17. Fujino T, Ishii Y, Takeuchi T, Hirasawa K, Tateda K, Kikuchi K, et al. Recovery of BMIPP uptake and regional wall motion in insulin resistant patients following angioplasty for acute myocardial infarction Circ J 2003; 67: 757-762.

18. Sugimoto K, Ito H, Iwakura K, Ikushima M, Kato A, Kimura R, et al. Intravenous nicorandil in conjunction with coronary reperfusion therapy is associated with better clinical and functional outcomes in patients with acute myocardial infarction. Circ J 2003; 67: 295-300.

19. Funabashi N, Ishida A, Yoshida K, Komuro I. Double aortic arch with a compressed trachea demonstrated by multislice computed tomography. Circulation 2004; 110: e68-e69.

20. Funabashi N, Kobayashi Y, Kudo M, Asano M, Teramoto K, Komuro I, et al. New method for measuring coronary diameter by electronbeam computed tomographic angiography using adjusted thresholds determined by calibration with aortic opacity. Circ J 2004; 68: $769-$ 777.

21. Funabashi N, Misumi K, Ohnishi H, Watanabe M, Suzuki Y, Imai N, et al. Endoluminal perspective volume rendering of coronary arteries using electron-beam computed tomography. Circ J 2003; 67: 10641067 .

22. Funabashi N, Kobayashi Y, Rubin GD. Utility of three-dimensional volume rendering images using electron-beam computed tomography to evaluate possible causes of ischemia from an anomalous origin of the right coronary artery from the left sinus of Valsalva. Jpn Circ J 2001; 65: 575-578

23. Funabashi N, Rubin GD. Direct identification of patency achieved by a bi-directional Glenn shunt procedure: Images by volume rendering using electron-beam computed tomography. Jpn Circ J 2001; 65: 457-461.

24. Pan T, Lee TY, Rietzel E, Chen GT. 4D-CT imaging of a volume influenced by respiratory motion on multi-slice CT. Med Phys 2004; 31: 333-340.

25. Nehmeh SA, Erdi YE, Pan T, Yorke E, Mageras GS, Rosenzweig KE, et al. Quantitation of respiratory motion during 4D-PET/CT acquisition. Med Phys 2004; 31: $1333-1338$.

26. Hirai T, Korogi Y, Ono K, Uemura S, Yamashita Y. Preoperative embolization for meningeal tumors: Evaluation of vascular supply with angio-CT. Am J Neuroradiol 2004; 25: 74-76.

27. Hunink MG, Gazelle GS. CT screening: A trade-off of risks, benefits, and costs. J Clin Invest 2003; 111: 1612-1619.

28. Knollmann F, Pfoh A. Coronary artery imaging with flat-panel computed tomography. Circulation 2003; 107: 1209. 